University of Nebraska - Lincoln

DigitalCommons@University of Nebraska - Lincoln

\title{
A Method for Estimating Fractal Dimension of Tree Crowns from Digital Images
}

\author{
Dongsheng Zhang \\ University of Nebraska-Lincoln \\ Ashok Samal \\ University of Nebraska-Lincoln, asamal1@unl.edu \\ James R. Brandle \\ University of Nebraska-Lincoln, jbrandle1@unl.edu
}

Follow this and additional works at: https://digitalcommons.unl.edu/csearticles

Part of the Computer Sciences Commons

Zhang, Dongsheng; Samal, Ashok; and Brandle, James R., "A Method for Estimating Fractal Dimension of Tree Crowns from Digital Images" (2007). CSE Journal Articles. 97.

https://digitalcommons.unl.edu/csearticles/97

This Article is brought to you for free and open access by the Computer Science and Engineering, Department of at DigitalCommons@University of Nebraska - Lincoln. It has been accepted for inclusion in CSE Journal Articles by an authorized administrator of DigitalCommons@University of Nebraska - Lincoln. 


\title{
A Method for Estimating Fractal Dimension of Tree Crowns from Digital Images
}

\author{
Dongsheng Zhang, ${ }^{1}$ Ashok Samal, ${ }^{1}$ and James R. Brandle ${ }^{2}$ \\ 1. Department of Computer Science and Engineering \\ 2. School of Natural Resources Science \\ University of Nebraska-Lincoln, Lincoln, Nebraska, USA \\ Corresponding author - A. Samal, email samal@cse.unl.edu
}

\begin{abstract}
A new method for estimating fractal dimension of tree crowns from digital images is presented. Three species of trees, Japanese yew (Taxus cuspidata Sieb \& Zucc), Hicks yew (Taxus $\times$ media), and eastern white pine (Pinus strobus L.), were studied. Fractal dimensions of Japanese yew and Hicks yew range from 2.26 to 2.70. Fractal dimension of eastern white pine range from 2.14 to 2.43 . The difference in fractal dimension between Japanese yew and eastern white pine was statistically significant at 0.05 significance level as was the difference in fractal dimension between Hicks yew and eastern white pine. On average, the greater fractal dimensions of Japanese yew and Hicks yew were possibly related to uniform foliage distribution within their tree crowns. Therefore, fractal dimension may be useful for tree crown structure classification and for indexing tree images.
\end{abstract}

Keywords: tree crown structure, foliage distribution, fractal scale, fractal surface area, fractal geometry

\section{Introduction}

Fractal geometry has had a major impact on modeling and analysis in the natural and physical sciences. A class of fractals, L-systems and stochastic L-systems, has been successfully used to model natural plants.,15,16 Mandelbrot has demonstrated that many fractal surfaces are generalizations of random walks and Brownian motion. ${ }^{7}$ Pentland has presented evidence that most natural surfaces are spatially isotropic fractals. ${ }^{12}$ Furthermore, the intensity images of these surfaces are also fractal. Fractals have been used in computer vision to model and recognize natural scenes. ${ }^{2,5,13,18}$ Keller et al. have used fractal dimension as a scale insensitive ruggedness measure by which they described silhouettes of tree lines and mountain lines. ${ }^{4}$ Fractal dimension has been shown to be a measure of toughness in metals ${ }^{8}$ and useful in modeling intercepted snow accumulation in for- 
ests. ${ }^{14}$ The fractal dimension has also been used together with other features for image texture description and segmentation. ${ }^{1,5,11}$ In forestry research, Zeide and Pfeifer have found that fractal dimension of tree crowns can be used as an indicator of tolerance, crown class and foliage distribution within a tree crown. ${ }^{19}$ Mandelbrot used fractals to model trees and analyze their structure. ${ }^{7}$ Morse et al. used the fractal dimension of vegetation to correlate the size of insects that live on it. ${ }^{10}$

Various ways of estimating fractal dimension have been used in the past. $6,7,9$ Several attempts have been made to estimate the fractal dimension from two-dimensional images. However, the estimates tell us little about the three-dimensional structure of the objects such as tree crowns. A method for the estimation of fractal dimensions of tree crowns has been developed by Zeide and Pfeifer. ${ }^{19}$ Unfortunately, the requirements of biomass measurement in this method make it inconvenient to use for practical purposes. The objective of this study was to develop a new method to estimate the fractal dimension of tree crowns from two-dimensional images, and to relate the fractal dimension of tree crowns to their internal structures.

\section{Fractal Basics}

A fractal is by definition a set for which the Hausdorff Besicovitch (fractal) dimension strictly exceeds the topological dimension. ${ }^{7}$ Unlike spatial dimensions in classical geometry, fractal dimensions may be real numbers. Ideal objects like straight lines, rectangles, and circles have integral spatial dimensions. In contrast, the spatial dimension of natural objects such as coast-lines, mountain outlines, boundary of leaves and snowflakes, is often greater than one. This dimension is also called the "fractal" dimension. ${ }^{7}$ The fractional part of the dimension portrays the degree of perturbation from the ideal line. Similarly, the fractal dimensions of natural surfaces such as clouds, mountains and tree crowns are greater than two.

Fractal geometry offers very important tools for describing and analyzing irregularity which can be classified as new regularity, seemingly random but with precise internal organization. ${ }^{17}$ Most of the measurements in forestry such as crowns, roots, soil, and landscapes are discontinuous, jagged and fragmented, and are difficult to estimate accurately. ${ }^{6}$ Fractal geometry can provide a useful tool in this regard.

Fractal geometry explicitly uses the concept of observation scale in building an object. An object is generated by repeating a process using a sequence of scales. However, all scales in a fractal are considered equivalent and objects at different scales are indistinguishable. This feature of fractals, called "self-similarity," is perhaps their most important property.

In fractal theory, length of a curve such as a coast line is a function of the measurement unit. ${ }^{7,9}$ If the length is measured using a range of scales, the relationship between the two can be determined and the fractal dimension of the object can be estimated. Determination of the fractal dimension of objects with greater complexity is often harder. 6,19 


\section{Estimation of Fractal Dimension of Tree Crowns from Digital Images}

A new method for estimation of fractal dimension of a tree crown is derived in this section. We use some results described by Zeide and Pfeifer ${ }^{19}$ and Peleg et al. ${ }^{11}$ Interested readers may see details of some of these steps in the above references.

Given a fractal curve such as a coast line, the length of the curve is given by:

$$
\mathrm{L}_{\xi}=a \xi^{1-D_{1}}
$$

where $\mathrm{L}_{\xi}$ is the length of the curve at scale $\xi$, i.e. the length of the yardstick used for measurement. $D_{1}$ is the fractal dimension of a one-dimensional object and $a$ is a constant.

Similarly the area for a fractal surface such as a tree crown is given by:

$$
\mathrm{A}_{\xi}=c \xi^{2-D_{2}}
$$

where $A_{\xi}$ is the fractal surface area at scale $\xi, c$ is a constant and $D_{2}$ is the fractal dimension of a tree crown.

Taking the natural logarithm on both sides of Equation (2), we have:

$$
\ln A_{\xi}=\left(2-D_{2}\right) \ln \xi+\ln c .
$$

This provides a mechanism to compute the fractal dimension of the tree crown. If the area of the crown $\left(A_{\xi}\right)$ is measured at different scales $(\xi)$, the slope of a line in a plot of $\ln A_{\xi}$ versus $\ln \xi$ would approximate $2-D_{2}$ from which we can easily compute the fractal dimension $D_{2}$. However, computation of surface area of the tree crown is a nontrivial task. Zeide and Pfeifer ${ }^{19}$ used biomass to estimate foliage area. A method to estimate the dimension using a digital image is used in this approach.

Using the self-similarity and symmetry properties, crowns of many evergreen species can be represented as two identical fractal surfaces. Thus an image can be used to capture the properties of the half tree crown surface. Without loss of generality, we use $A_{\xi}$ to represent the area of this half.

\subsection{Measuring fractal surface area from a digital image}

We use an approach similar to one proposed by Mandelbrot ${ }^{7}$ for measuring the lengths of irregular coast-lines. The algorithm is based on the following observation. Consider all points with distances to coast-lines of no more that $\xi$. These points form a strip of width $2 \xi$. The length $L_{\xi}$ of the coast line is the area of the strip divided by width, $2 \xi$.

To compute the fractal surface area of one side of a tree crown, the above approach for estimating coast-lines is adapted. In this extension from curve to fractal surface, we cover the fractal surface of the tree crown using $2 \xi \times 2 \xi \times 2 \xi$ cubic units with the center point of each cubic unit on the surface of the tree crown. The 
surface area of the tree crown shown in digital images is then the volume occupied by the cubic units divided by $2 \xi$.

The volume of each cubic unit is proportional to the number of pixels inside the $2 \xi \times 2 \xi$ square centered at $(i, j)$ which satisfy the following property:

$$
g(i, j)-\xi<g(i, j)<g(i, j)+\xi
$$

where $g(i, j)$ is the intensity at location $(i, j)$ in the digital image. Thus the volume of the cubic unit corresponding to the point $(m, n)$ in the image is:

$$
V_{\xi}=(m, n) \propto \sum_{i=-\xi}^{\xi} \sum_{j=-\xi}^{\xi} \mu(i, j)
$$

where

$$
\mu(i, j)=\left\{\begin{array}{l}
1 \text { if } g(i, j)-\xi<g(i, j)<g(i, j)+\xi \\
0 \text { otherwise }
\end{array}\right.
$$

The algorithm for estimation of fractal dimension of a tree crown is summarized below:

(1) For each scale $\xi=1,2, \ldots, 50$

For each cubic unit over a tree crown part of the image:

Determine the volume of each cubic unit $\mathrm{V}_{\xi}(m, n)$ using Equation (5).

Calculate the total volume of cubic units covering the tree crown surface at scale $\xi$.

$$
V_{\xi}=\Sigma_{m, n} V_{\xi}(m, n)
$$

Determine the fractal surface area of the tree crown in the digital image at scale $\xi$.

$$
A_{\xi}=\frac{V_{\xi}}{2 \xi}
$$

(2) Determine the slope of the line $\gamma$ given in Equation (3) using the Least Square method for $A_{\xi}$ and $\xi$ plot.

(3) Calculate the fractal dimension, $D_{2}$, of a tree crown as follows:

$$
D_{2}=2-r \text {. }
$$

It should be noted that since we use a linear sampling rather than log sampling of $\xi$, the measurement scale, the line fitting procedure is weighted towards the larger scales since there are more points there.

\section{Implication of the New Method for Estimation of Fractal Dimension of a Tree Crown}

The new method derived in the previous section is based on fractal geometry theory which uses the concept of observation scales in building an object [Equa- 
tion (1)] and is an extension of the classical approach for estimating fractal dimension of a one-dimensional object [Equation (2)]. Obviously pixel values in the image of a tree crown are functions of many factors such as light condition, camera position and the internal structure of a tree crown. The focus of the derivation of the method for estimating fractal surface area [Equations (4)-(6)], therefore, is given to the relative value of each pixel as related to other pixel values within each cubic unit at each measuring scale. Under certain fixed environmental conditions such as light condition and camera position and assuming the greenness of a tree crown is evenly distributed, the relative value of each pixel [Equation (4)] or the volume of each cubic unit [Equations (5) and (6)] reasonably reflects the fractal surface condition or tree crown structure. On the calm days when the videos were taken, the method derived in this paper for estimating fractal surface area, which is the foundation for calculating fractal dimension, should approximately reflect tree crown structures. Unlike other methods for estimating fractal dimension from two-dimensional images $6,7,9$ the relative value of each pixel associated with measuring scales plays an important role in calculating fractal surface area and fractal dimension. In comparison to the method developed by Zeide and Pfeifer, ${ }^{19}$ the method derived in this paper is relatively easy to use for practical purposes.

\section{Materials and Methods}

\subsection{Study site and tree species}

Three common species of trees, Japanese yew (Taxus cuspidata Sieb \& Zucc), Hicks yew (Taxus $\times$ media), and eastern white pine (Pinus strobus L.), were grown at the Landscape Services Nursery, University of Nebraska-Lincoln. These trees were five to eight years old, $0.5 \mathrm{~m}$ to $1 \mathrm{~m}$ tall, and are commonly used in landscape plantings in eastern Nebraska.

\subsection{Image acquisition, feature extraction and tree crown recognition}

Color images of trees in the field were recorded on a video tape using a SONY Video Cameral Recorder (Model No. CCD-V5000). The images were grabbed into the storage of a UNIX SUN SPArcstation running SUNOS 5.4 using a high quality frame grabber (RasterOps) capable of acquiring the images at 24 bits true color.

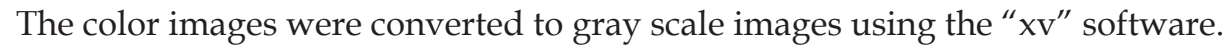

Feature extraction is the process of computation of relevant features that result in quantitative information of interest. A statistical texture analysis approach was used in this study and a set of texture measures were derived from images. These measures in conjunction with standard image analysis techniques were used to separate tree crowns from their background. Details of feature extraction and tree crown recognition are documented in the work by Zhang et al. ${ }^{20}$ Some examples of the original images are shown in Figure 1. Example of tree crowns that have been separated from their backgrounds and used for estimation of fractal dimensions are shown in Figure 2. 


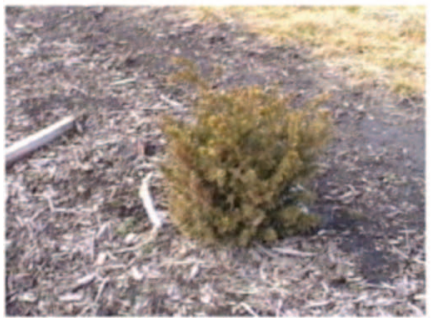

(a) Japanese yew

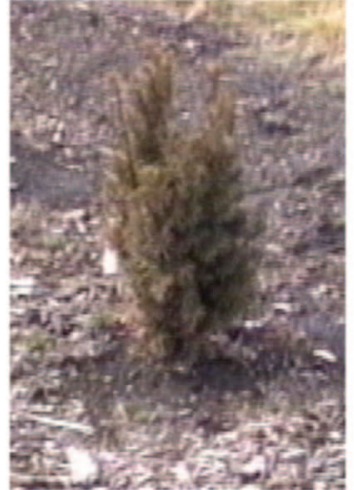

(b) Hicks yew

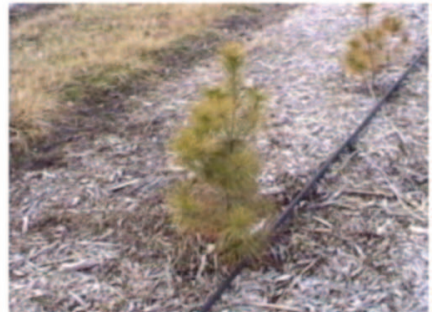

(c) Eastern white pine

Figure 1. Sample images for the three tree species.

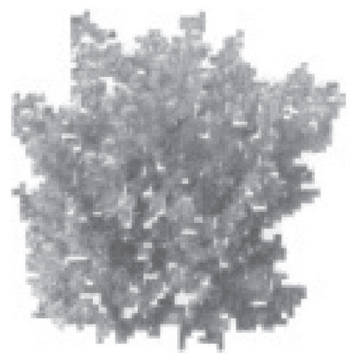

(a) Japanese yew

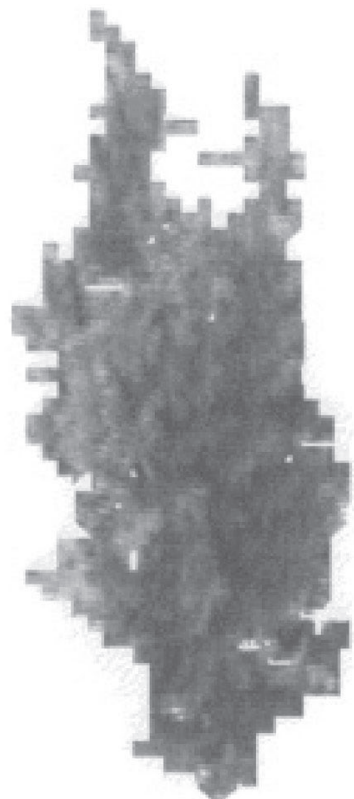

(b) Hicks yew

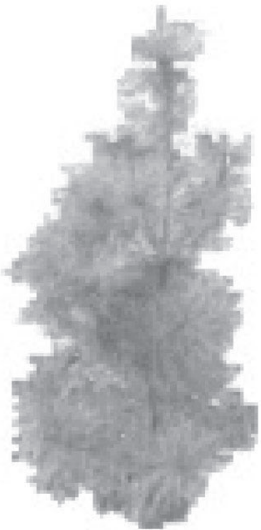

(c) Eastern white pine

Figure 2. Separation of tree crowns from the background.

\subsection{Results and discussions}

Fourteen (14) images for each tree species were used for estimation of fractal dimension. The images for each species were obtained using 14 different trees. In general, Japanese yew had crowded leaves and relatively regular crown structure 
(a) Japanese Yew

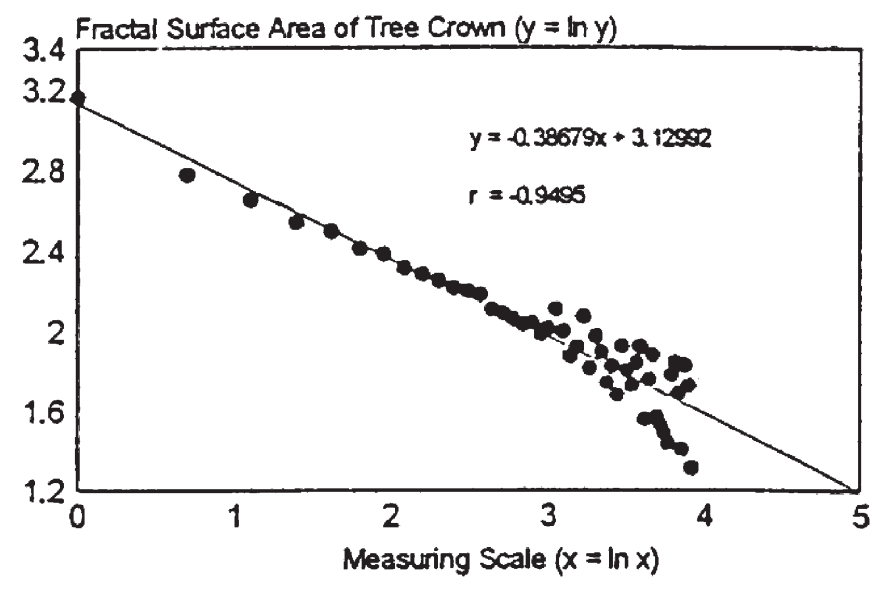

(b) Hicks Yew

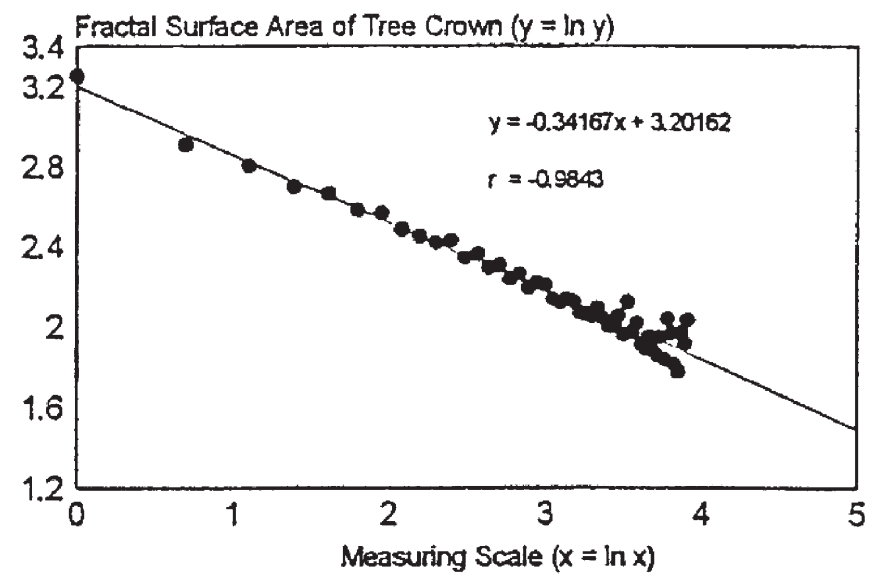

(c) Eastern white pine

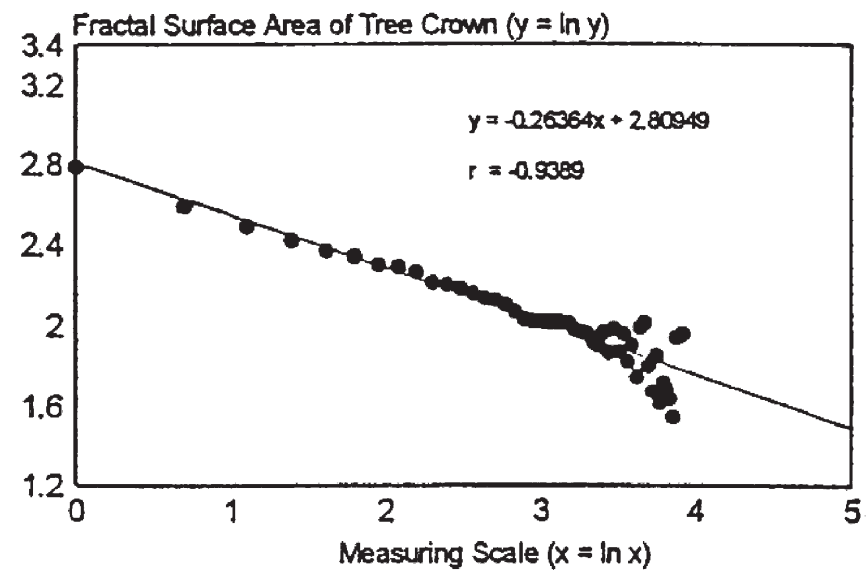

Figure 3. Relationship between scale and the crown surface area ( $\xi$ versus $A_{\xi}$ ). 
Table 1. Average fractal dimension for the tree species.

\begin{tabular}{lccc}
\hline $\begin{array}{l}\text { Name of } \\
\text { Tree Species }\end{array}$ & $\begin{array}{c}\text { Number of } \\
\text { Images }\end{array}$ & $\begin{array}{c}\text { Average Fractal } \\
\text { Dimension }\end{array}$ & $\begin{array}{c}\text { Standard } \\
\text { Error }\end{array}$ \\
\hline Japanese yew & 14 & 2.45 & 0.03 \\
Hicks yew & 14 & 2.42 & 0.04 \\
Eastern white pine & 14 & 2.24 & 0.03 \\
\hline
\end{tabular}

Table 2. Results from General Linear Models (ANOVA) to test differences in the fractal dimensions of the three tree species.

\begin{tabular}{lcllcl}
\hline $\begin{array}{c}\text { Tree } \\
\text { Species (1) }\end{array}$ & $\begin{array}{c}\text { Tree } \\
\text { Species (2) }\end{array}$ & $\begin{array}{l}\text { Dependent } \\
\text { Variable }\end{array}$ & $\begin{array}{c}\text { Mean } \\
\text { Square }\end{array}$ & $\begin{array}{c}\text { F } \\
\text { Value }\end{array}$ & $\begin{array}{c}\text { Probability } \\
\text { Value }\end{array}$ \\
\hline Japanese yew & Eastern white pine & Fractal Dimension & 0.33223 & 25.33 & 0.0001 \\
Hicks yew & Eastern white pine & Fractal Dimension & 0.12275 & 6.57 & 0.0051 \\
Japanese yew & Hicks yew & Fractal Dimension & 0.00654 & 0.39 & 0.5373 \\
\hline
\end{tabular}

with foliage being uniformly distributed throughout tree crown volume (Figure 1). In contrast, eastern white pine had irregular tree structure with foliage being mainly located close to the tree crown surface. Hicks yew was similar to Japanese yew in tree crown structure but with a more upright vase shape (Figure 1).

The relationship between the fractal surface areas and the measuring scales for the three studied species in the digital images are presented in Figure 3. The figures show the relationship for sample images from each species and are not composites over all the images. The linear relationship between fractal surface areas and the measuring scales in digital images conform with the mathematical derivation given in Equation (3).

The average fractal dimensions of the studied species vary from 2.45 for Japanese yew to 2.24 for eastern white pine (Table 1). It is not surprising to find that the average fractal dimension of Hicks yew is close to that of Japanese yew (Table 1) because they have similar crown structures. The greater standard error of fractal dimension for Hicks yew compared with others is possibly related to greater variation in shape from one tree to another (Table 1). The difference in fractal dimension between Japanese yew and Hicks yew was not statistically significant (Table 2). The difference in fractal dimension between Japanese yew and eastern white pine was statistically significant at 0.05 significance level, as was the difference in fractal dimension between Hicks yew and eastern white pine (Table 2).

In general, the difference in fractal dimension between tree species reflects the difference in tree crown structure. The smaller value of fractal dimension indicates that the foliage of the tree crown is located on the crown periphery and its mass and surface are proportional to the surface of the convex hull. ${ }^{19}$ On the other hand, a large value of fractal dimension implies that foliage was uniformly distributed throughout the crown volume. ${ }^{19}$ According to our measurements, fractal 
dimension for Japanese yew ranges from 2.26 to 2.70 , and the fractal dimension for the eastern white pine varies from 2.14 to 2.43. The effect of the shape of tree crown on estimation of fractal dimension is not immediately obvious and further analysis is needed in this area.

Furthermore, the experiments were conducted under controlled conditions. For example, the trees were isolated and were approximately the same age, grown under controlled conditions in a nursery. In uncontrolled environments, the trees will not be as well behaved; trees may be clustered making their separation harder, they may have damage due to harsh weather and climatic conditions or disease. Some issues that can be addressed include nonuniform illumination, and scale. A histogram equalization step can be performed to correct for different illumination conditions. Similarly, the different size of the objects can be addressed by normalizing the scale, if necessary.

Another contribution of this research is that the approach is used to compute the fractals of real trees, and not synthetic trees. Itis assumed that the growth of the trees is uniform. While trees of the same species cannot be expected to have the exact growth patterns, most trees follow regular patterns of growth. While they were grown under controlled conditions, all plants are not exactly the same. There are significant variations among the trees. Indeed the sample trees of each species had significant variation, but the analysis showed that the intra-class variation is statistically different from inter-class variation. Again, in this paper we have developed a computationally efficient method to compute the fractal dimensions of tree crowns and demonstrated its effectiveness in classification in a limited test set. Some practical issues need to be addressed before the results can be used in a broad and practical problem.

\section{Conclusion}

Several attempts have been made to estimate fractal dimension of three-dimensional surface area from digital images. It has been known that the two surface method provided by Zeide et al. is the first and so far the only workable method for determining the fractal dimension of real tree crowns as opposed to their two-dimensional projections. ${ }^{19}$ However, this method requires traditional biomass measurements. The proposed method in this study for estimation of fractal dimension of tree crowns from digital images is easy to use in practice. In this approach, the image of a tree is obtained via a digital camera, aerial photography or even high resolution satellite sensors (e.g. IKONOS). After a sequence of image processing operations, the fractal dimension of the tree crown is estimated using the algorithm described in this paper. In general, the results of this study complement the previous finding ${ }^{19}$ and show that fractal dimensions of tree crowns can serve as an index for classifying tree crown structure.

We have shown that the fractal dimension can be used to classify a small number of species. This work can be extended to explore if they can be used in a classification system with significantly large number of classes, as it is likely in 
a practical setting. The fractal dimension of tree crowns is limited to the narrow range between 2 and 3. Thus the discrimination power may be somewhat limited. However, this approach can still be used to group several species with close fractal dimensions into broad classes to obtain a coarse classification. A potential application of this approach is to inventory trees in urban landscapes. Both gradual loss of trees over time and sudden loss due to severe weather events can be estimated efficiently using this approach. For this to be successful, more extensive experimentation is needed and is left as a part of our future work. In the absence of large human resources to inventory trees in large geographic areas, it may be better to get an approximate inventory of trees than none. In addition, even a coarse classification can lead to rough, but quick estimate of carbon sequestration amounts, by using the volume of trunks and branches as an approximation for amount of biomass.

Acknowledgment - This study was supported by funds from the McIntyreStennis Cooperative Forestry Research Program.

\section{References}

1. B. B. Chaudhuri and N. Sarkar. 1995. Texture segmentation using fractal dimension, IEEE Trans. Patt. Anal. Mach. Intell. 17(1): 72-77.

2. S. S. Chen, J. M. Keller and R. M. Crownover. 1990. Shape from fractal geometry, Artif. Intell. 43: 199-218.

3. D. J. Holliday and A. Samal. 1995. Object recognition using L-system fractals, Patt. Recogn. Lett. 16: 33-42.

4. J. M. Keller, R. M. Crownover and R. Y. Chen. 1987. Characteristics of natural scenes related to the fractal dimension, IEEE Trans. Patt. Anal. Mach. Intell. PAMI-9(5): 621-627.

5. J. M. Keller, R. M. Crownover and R. Y. Chen. 1989. Texture description and segmentation through fractal geometry, Comput. Vis. Graph. Imag. Process. 45: 150-166.

6. N. D. Lorimer, R. G. Haight and R. A. Leary. 1994. The fractal forest: fractal geometry and applications in forest science, General Technical Report NC-1770, Department of Agriculture, USA.

7. B. B. Mandelbrot. 1982. The Fractal Geometry of Nature (W. H. Freeman, San Francisco).

8. B. B. Mandelbrot, D. E. Passoja and A. J. Paullay. 1984. Fractal character of feature surfaces of metals, Nature 308: 721-722.

9. A. L. Mehaute. 1991. Fractal Geometries: Theory and Applications, translated by Dr. J. Howlett (CRC Press, London).

10. D. R. Morse, J. H. Lawton, M. M. Dodson and M. H. Willamson. 1985. Fractal dimension of vegetation and distribution of arthropod body lengths, Nature 314: 731-733.

11. S. J. Peleg, J. Naor, R. Hartley and D. Avnir. 1984. Multiple resolution texture analysis and classification, IEEE Trans. Patt. Anal. Mach. Intell., PAMI-6(4): 518-523.

12. A. Pentland. 1984. Fracta-based description of natural scenes, IEEE Trans. Patt. Anal. Mach. Intell. PAMI-6(6): 661-674. 
13. A. Pentland. 1986. Perceptual organization and representation of natural form, Artif. Intell. 28: 293-331.

14. J. W. Pomeroy and R. A. Schmidt. 1993. The use of fractal geometry in modelling intercepted snow accumulation and sublimation, Proc. 50th Anniversary Meeting of Eastern Snow Conf., p. 1-9.

15. P. Prusinkiewicz and A. Lindenmayer. 1990. The Algorithmic Beauty of Plants (SpringerVerlag, NY).

16. A. Samal, B. Peterson and D. J. Holiday. 2002. Recognizing plants using stochastic Lsystems, J. Electron. Imag. 11(1): 50-58.

17. I. Stewart. 1988. A review of: the science of fractal images, Nature 336: 289.

18. R. Voss. 1986. Random fractal: characterization and measurement, in Scaling Phenomena in Disordered Systems, editors R. Pynn and A. Skjeltorp (Plenum Press, NY).

19. B. Zeide and P. Pfeifer. 1991. A method for estimation of fractal dimension of tree crowns, Forest Sci. 5: 1,253-1,266. 20. D. J. Zhang, J. Brandle and A. Samal. 2006. Texture as the basis for individual tree identification, Inform. Sci. 176(5): 565-576.

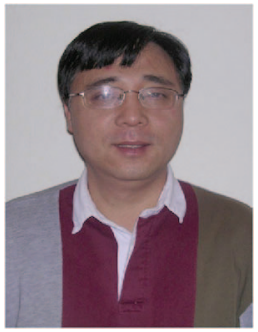

Dongsheng Zhang received the M.S. degrees in forestry and computer science from the University of Nebraska-Lincoln, in 1994 and 1996, respectively. He has been working as a senior consultant and software engineer for First Data Corporation, Verizon Telecommunications, MCI Telecommunications, ATT Research laboratory, and URL Integration Inc since 1995. Currently he is a principal consultant with URL Integration Inc, Colorado Springs, CO.

His research interests include image processing and analysis, pattern recognition, object-oriented programming and software architecture.

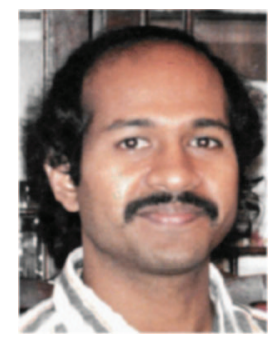

Ashok Samal received his Bachelor of Technology degree in computer science from Indian Institute of Technology at Kanpur, India. He received his Ph.D. in computer science from the University of Utah. He is currently with the Department of Computer Science and Engineering at the University of Nebraska-Lincoln as an Associate Professor.

His research interests are in image understanding, document analysis, geospatial computing, and distributed computation. He has published over 70 papers in these areas in international journals and conferences. 


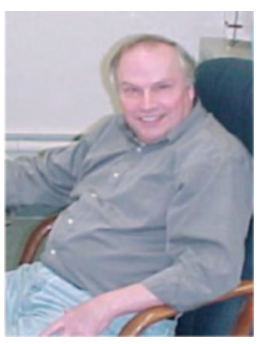

James R. Brandle is Professor of Forestry at the University of Nebraska-Lincoln in the area of shelterbelt ecology. His work focuses on the effects of wind protection on crop production and yields, the mechanisms of wind flow in the vicinity of a windbreak as affected by windbreak structure and the ecological benefits of woody plants to the agricultural ecosystem. He has published widely on the benefits of windbreaks and is the editor of the book Windbreak Technology, the standard text on windbreaks. His teaching focuses on forest management, agroforestry and woody plant growth and development. 